\title{
$\underline{\text { Reducing Parenting Stress in Families With Irritable Infants }}$
}

By: Maureen R. Keefe, Kristine A. Kajrlsen, William N. Didley, Marie L. Lobo, and Anne Marie Kotzer

Keefe MR, Kajrlsen KA, Lobo ML, Kotzer AM, Dudley WN. (2006). Reducing parenting stress in families with irritable infants. Nurs Res, 55(3), 198-205.

Made available courtesy of LIPPINCOTT WILLIAMS \& WILKINS:

http://journals.lww.com/nursingresearchonline/Pages/default.aspx

This format of the article is not the final published version.

$* * *$ Note: Figures may be missing from this format of the document

Abstract:

Background: Caring for an infant with unexplained, persistent crying is one of the most stressful events for new parents. Infant irritability, also referred to as infantile colic, occurs in 10$25 \%$ of all infants and is the most common parental concern reported in the first year of life.

Objective: The aim of this study was to evaluate the effectiveness of a home-based nursing intervention in reducing parenting stress in three groups of families with irritable infants, using data from a larger evaluation study.

Methods: A two-site, randomized clinical trial was conducted with 121 infants and their parents. Infants were randomized to a treatment or a control group. A third group of infants $(n=43)$ was added as a posttest-only comparison. The level of parenting stress was measured by the Parenting Stress Index (PSI) at baseline, after the 4-week intervention, and at the 8-week followup visit.

Results: A repeated measures analysis of variance (ANOVA) was used to compare the two groups across the three time points (baseline, immediately after the 4-week intervention, and at the 8-week follow-up visit). The results revealed a reduction in total parenting stress over time for both the treatment and control groups. Mothers in the treatment group reported reduced parenting stress on the parent-child dysfunctional interaction subscale $(\mathrm{p}=.04)$. Total parenting stress scores were found to be significantly higher for mothers in the posttest-only group ( $\mathrm{p}=$ .009).

Conclusion: Initial parenting stress levels were high in all participants. Parent feedback at the exit interview indicated that the nurse visits for data collection were also viewed as helpful in reducing the stress level associated with parenting these irritable infants. This home-based program was perceived as helpful in improving the interactions between parents and their irritable infants.

Key Words: infant colic; parenting stress

\section{Article:}

Caring for an infant with unexplained, persistent crying is one of the most stressful events in the lives of new parents. Infant irritability, also referred to as infantile colic, occurs in 10-25\% of all infants. It is characterized by inconsolable crying that usually begins at 1-2 weeks of age, with a peak in the crying time at 6 weeks to 2 months of age. The unexplained crying episodes often 
last 3-4 hours and can continue until 4-6 months of age. Although the persistent, unexplained crying episodes diminish over the first 3-6 months, the intensity and persistence of the crying behavior are extremely distressing to new parents. Adding to the potential for parenting stress is the lack of adequate information regarding the cause of infant irritability and effective strategies for managing the situation (Barr, St. James-Roberts, \& Keefe, 2001a, 2001b; Keefe, 1988).

The purpose of the larger research investigation from which the data of this study were derived was to evaluate the effectiveness of a home-based intervention for managing infant irritability through a randomized, two-site clinical trial. This article will focus on findings related to the effects of the intervention on parenting stress levels. The findings regarding the effects on infant crying behavior are reported elsewhere (Keefe, Lobo, Froese-Fretz, Kotzer, Barbosa \& Dudley, 2006).

Studies exploring the challenges of parenting a colicky or irritable infant are focused primarily on mothers as the primary caregivers in the early months of life. Papousek and von Hofacker (1998) reported a strong drain on the psychological and physical resources of mothers of infants with persistent crying. Others have described caring for an irritable or colicky infant as a crisis situation with ramifications for parent-infant relationships, marital relationships, and family dynamics in general (Humphrey \& Hock, 1989; Stifter \& Bono, 1998). Recurrent episodes of unexplained and inconsolable infant crying have been associated with feelings of inadequacy, depression, fatigue, and resentment on the part of the mother (Keefe \& Froese-Fretz 1991; Murray \& Cooper 2001; Thompson, Harris, \& Bitowski, 1986). In some cases, colic or persistent, unexplained crying has been associated with the shaken baby syndrome or identified as a trigger for infant abuse or other forms of parental dysfunction such as inattentiveness and neglect (Barr et al., 2001b; St. James-Roberts \& Plewis, 1996).

In two recent studies, researchers explored a behavioral approach to the management of colic. St. James-Roberts, Sleep, Morris, Owen, and Gillham (2001) evaluated the use of a behavioral program in the first 3 months to prevent infant crying and sleeping problems. Low compliance with the behavioral recommendations was revealed in journals. However, fewer parents in this treatment group sought help for crying and sleeping problems during the following 6 months. Investigators in Australia had limited success in reducing sleep problems at 2 months using a sleep intervention based on limited periods of controlled infant crying (Hisock \& Wake, 2002). A home-based intervention consisting of emotional support and parenting education for parents of drug-exposed infants resulted in fewer behavioral problems and a trend toward lower parental distress scores (Butz et al., 2001).

\section{Theoretical Framework}

In 1988, Keefe proposed a theoretical model that conceptualized infant colic from a developmental psychobiological perspective. Colic, referred to as irritable infant syndrome, is viewed as a delay or disturbance in the infant's sleep-wake cycling or an infant state regulation disorder. Infant state as popularized by Brazelton (1995) is conceptualized as a level of arousal continuum ranging from deep, quiet sleep to awake and aroused lusty crying. In the proposed model, the colicky or irritable infant has a disorganized or undeveloped sleep-wake cycle that underlies the excessive crying and difficulty initiating sleep (see Figure 1). The process underlying the observed behavioral manifestations is seen as a dysfunction in an infant's ability 
to self-regulate state or sleep-wake cycles based on central nervous system immaturity rather than gastrointestinal system disorder. This behavior instability may be exacerbated by parental inconsistency and environmental disruptions. From this framework, irritable infants are viewed as sensitive and are more easily overstimulated by busy chaotic environments. As infants become overwhelmed and fatigued, they cannot self-sooth or reduce their arousal level sufficiently to fall asleep. Parents, although very concerned, may be reinforcing the irritable behavior pattern by inconsistent attempts at strategies that are not contingent with the infant's unclear signals and erratic cues. Infant irritability, when viewed as a developmental state regulation disorder that disrupts the synchrony of the parent-infant dyad, necessitates an approach that provides support for the parents and modification of the infant's care and environmental routines during this early sensitive period (Keefe, 1988).

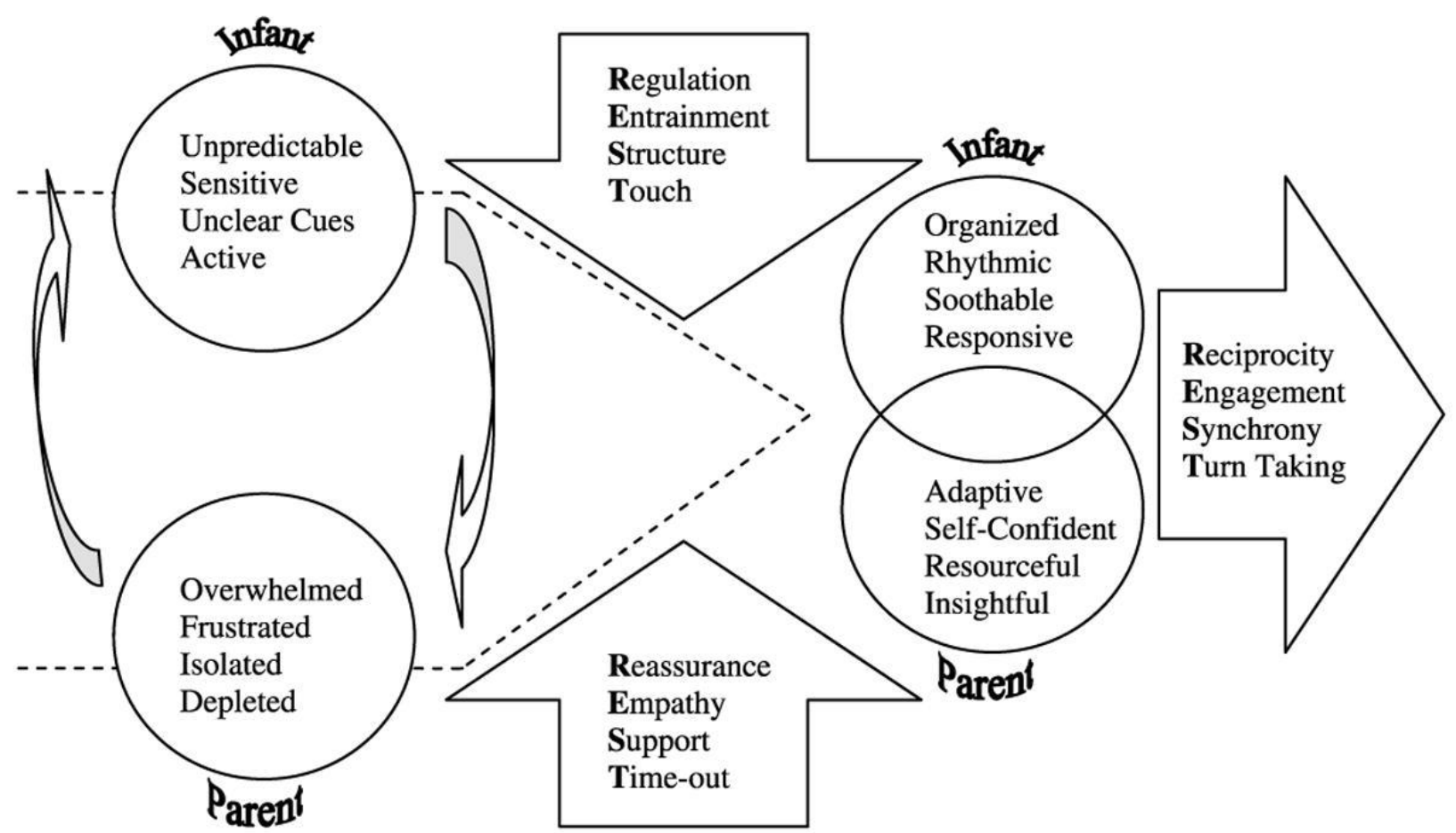

FIGURE 1. Theoretical model of infant irritability.

\section{Research Design and Methods}

A randomized, clinical trial was used to test the effectiveness of a home-based nursing intervention in (a) reducing infant crying, (b) increasing infant state stability (sleep-wake cycles), (c) improving the quality of the maternal-infant interaction, and (d) reducing parenting stress.

The findings reported in this article relate to the fourth outcome measure, and the data are a subset of a larger study. Other outcomes of the research project are reported in Keefe et al. (2006). Specifically, this aspect of the study was designed to test the following research hypothesis: Mothers who received the home-based nursing intervention for infant irritability will report less parenting stress than the mothers who did not receive the intervention.

A secondary aim was added to the study design after the initiation of recruitment efforts. This secondary aim was developed to compare the findings from the two randomized groups of infants with that of a third, posttest-only group that received only one house visit. This three-way 
analysis was included to evaluate the therapeutic effect of home visiting by a nurse and to include an older infant age cohort in the study.

\section{Participants}

The sample consisted of healthy, full-term infants with excessive unexplained irritability or colic and their parents (Keefe et al., 2006). Families with irritable infants were recruited through referrals from local pediatricians and from direct self-referral through study information in newsletters, local radio, brochures, and flyers posted in churches and stores. The study enrollment requirement was set at more than $2.5 \mathrm{hr} /$ day of unexplained crying over the past 2 weeks as a minimum. Infant age criteria was set at less than 6 weeks. Several families called with infants over this age cutoff. These infants were enrolled in a posttest-only group for post hoc comparison with the randomized sample (see Figure 2). The study received institutional review board approval. One hundred eighty-seven families were assessed for eligibility. After informed consent was obtained, 137 families were randomized to routine care or the home-based intervention program using a computer-generated random assignment to equalize groups by sites. A posttest-only group $(n=43)$ was formed of infants who did not meet the infant age criteria. These infants were enrolled at an older age (mean age $=10.4 \pm 2.1$ weeks) and were not randomized. This posttest-only group was added to the study to include these older infants and to provide an additional follow-up comparison dataset from families who did not receive repeated home visits.

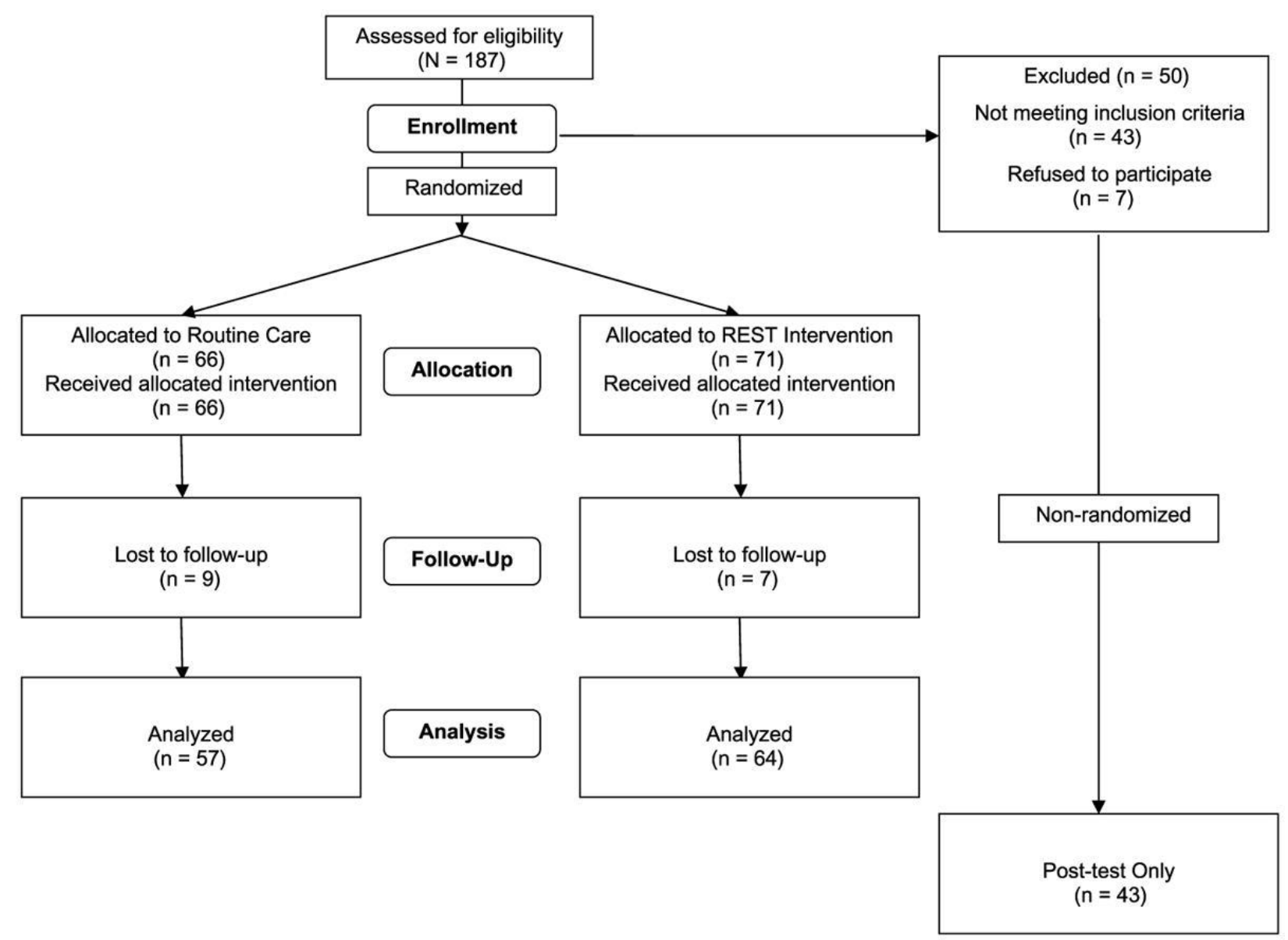

FIGURE 2. Enrollment flowchart. 


\section{Intervention}

The REST (reassurance, empathy, support, and time-out) Routine program (Barr et al., 2001a; Keefe, Froese-Fretz, \& Kotzer, 1997) is a home-based intervention model where a nurse works in partnership with the family. The program consisted of four home visits that occurred at weekly intervals after enrollment and baseline data collection. The intervention was individualized and delivered by a master's-prepared pediatric nurse specialist who had been trained in the intervention model and underlying concepts. Each home visit was approximately $1 \mathrm{hr}$ in length and included infant behavior assessments and demonstrations. Educational materials, a video entitled "Fussy Babies and Frantic Families," and accompanying worksheets and observation guides were left in the home to be reviewed and shared with the infant's father if he was not available for the visit. Pattern recognition, parental support, and reassurance were included as components of the REST Routine intervention program for irritable infants and their families (Keefe, Barbosa, Froese-Fretz, Kotzer, \& Lobo, 2005; Keefe et al., 1997).

These four REST concepts were individualized and applied in some of the following ways.

Reassurance was focused on two areas: the infant's health and the parent's competence. A review of the infant's general health status confirmed that illness and an identifiable source of pain had been ruled out based on a thorough physical examination of the infant. Parents were also provided reinforcement that they are competent and capable in their parenting roles. Reassurance was given also that excess irritability is a time-limited phenomenon with no known adverse sequelae.

Empathy was provided through listening and acknowledging the challenge of parenting these high-need and high-maintenance infants. Sharing the experiences of others helped overcome feelings of isolation and also normalized the range of feelings expressed by these parents. Through listening and avoiding simplistic advice, the nurse acknowledged the plight of the parents, including sleep deprivation and lack of positive feedback or parenting gratification.

Support involved serving as an advocate and resource for parents in obtaining assistance and creating a support network. Family and friends were mobilized to provide potential relief for the family. Support in the form of direct care providers for the irritable infant as well as others available to share experiences were identified. Professional support and resources, such as office or home visits, counseling, and other professional referrals, were discussed. Easy access to these resources and their availability by telephone or personal contact were identified as keys to avoiding isolation and parental overload.

Time-out from parenting was planned and reinforced as a basic self-care need. A specific period of time (at least 1hr) was scheduled into each day. This time was designated as a break or time off from direct caregiving, to be used for rest and renewal. The mother or caregiver was out of the range of infant crying and engaged in something that she enjoyed. A specific plan for coverage at a specific time each day was negotiated within the family or immediate social support network.

A more complete description of the home-based nursing intervention is contained in a separate publication (Keefe et al., 2005). 


\section{Instrument}

The Parenting Stress Index (PSI) is used to measure facets of stress in the parent-child system and to identify dysfunctional or at-risk parenting by assessing the behavioral characteristics of the child, the parents' sense of parenting competence, the quality of the marital relationship, and the availability of social support (Abidin, 1995). This theoretical model of the determinants of dysfunctional parenting guided the construction of the PSI with the purpose of surveying a diverse range of potential influences on parenting practices (Reitman, Currier, \& Stickle, 2002). The initial 120-item scale was normed on a variety of parents of children ranging in age from 1 month to 12 years (Abidin, 1995). Normative data displays and percentile rankings were derived for the total PSI and each PSI subscale and were based on the raw score frequency distributions of responses from mothers $(\mathrm{N}=800)$ in the normative sample (Abidin, 1995).

In the current study, the PSI Short Form (PSI-SF), an abbreviated 36-item scale derived from the full-length PSI, was used to reduce the response burden on the parent (Abidin, 1995; Reitman et al., 2002). This time-efficient tool takes approximately $15 \mathrm{~min}$ for the parent to complete and was used to minimize the data collection encounter and allow time for additional measures. The PSI-SF contains the following three subscales: difficult child (DC), parental distress (PD), and parent-child dysfunctional interaction (P-CDI). Each of the three subscales contains 12 items, which are rated on a Likert scale from 1(strongly disagree) to 5 (strongly agree). All items on the PSI-SF responses were recoded so that higher scores indicate higher levels of parenting stress. A score of 3 indicates not sure. Subscale scores range from 12 to 60; the total score ranges from 36 to 180 (Reitman et al., 2002).

A factor analysis to assess construct validity was conducted using a subsample of 121 participants from the current study. All 36 items were retained in the three subscales that emerged, and there was broad agreement between the factor loadings in this sample and the original factor structure identified by Abidin (1995). Twelve items loaded on Factor I and included all 12 items from Abidin's DC subscale. The 13 items that loaded on Factor II contained 10 of the 12 items from the PD subscale. Eleven items loaded on Factor III and comprised 9 of 12 items from Abidin's P-CDI subscale. The Cronbach's alpha statistic used on the subsample of 121 mothers indicated good internal consistency reliability, ranging from .83 to .88 . The total scale internal consistency with a Cronbach's alpha was .92 (Table 1). 


\section{Number}

Difficult child

Parental distress

Parent-child dysfunctional interaction

Total scale
12

13

11

36
$.58 \quad .88$

.62

.83

.61

.85

$-\quad .92$

TABLE 1. Parenting Stress Index-Short Form 36-Item Test-Retest and Cronbach Alpha Statistic

The PSI and PSI-SF have been used extensively to assess the parent-child dyad in a variety of clinical and research situations (Thomas, Renaud, \& DePaul, 2004; Uzark \& Jones, 2003; Youngblut et al., 2001).

\section{Data Collection Methods}

Complete data sets were obtained from 121 families enrolled in the randomized, two-site clinical trial conducted in Denver, CO, and Charleston, SC. A computer-generated randomization log of the ID numbers was created by the statistical staff to maintain treatment group equity at each data collection site. After telephone screening and intake, the research office staff enrolled, assigned the next available ID number, and coded for group assignment. Then, they set up the data collection and intervention visits if indicated. Consistency of the intervention and the data collection methodology was monitored across the sites with initial joint training sessions, frequent site visits, and periodic reliability checks. A separate trained evaluation team of data collectors obtained the parenting stress measurements at baseline, immediately after the 4-week intervention program, and at the 8-week follow-up visit in the home. The data collectors were pediatric or maternal-child nurses who were blind to the group assignment but were not trained in any of the concepts or principles of the intervention program. The families enrolled in the posttest-only group $(n=43)$ had infants older than 6 weeks of age. However, they met all the other sample selection criteria. These families were not randomized and had only one home visit for the collection of data. These data included demographic data and measures of infant fussiness, infant sleep, parenting stress, and maternal infant interaction (Keefe et al., 2006).

\section{Data Analysis}


A repeated measures analysis of variance (ANOVA) was used to compare total PSI-SF scores and scores for each subscale for the control, treatment, and posttest-only groups across the three time points (baseline, 4 weeks, and 8 weeks follow-up), and a one-way ANOVA was used to compare these values among all three groups (control, treatment, and posttest-only) at the final time point ( 8 weeks). Outcomes were analyzed using the SAS statistical software (SAS Institute Inc., Cary, NC). In all computations of the PSI-SF subscales, participants were allowed to miss approximately $20 \%$ of items and still produce a score.

Percentile rankings of the raw PSI scores within this study were also compared with the standardized percentile ranks developed for clinical use and data interpretation. The development of these ranks has been described previously (Abidin, 1995). For the PSI-SF, a total score at or above the 90th percentile indicates that the respondent's score is equal to or greater than the scores of $90 \%$ of the participants in the normative sample (Uzark \& Jones, 2003).

\section{Results}

The control group consisted of 57 (47\%) families, and the treatment group consisted of 64 (53\%) families who received the 4-week intervention to assist with their infant's fussy behavior. Parent demographic profiles revealed a predominately White sample (control group, 70.2\%; treatment group, $81 \%$ ). The mothers of the infants were in their early 30s (controls, $31.1 \pm 5$ years; treatment group, 31.2 \pm 6 years) with some college education (3.6 and 2.9 years post high school, respectively). Mean infant age at enrollment was 5 weeks of age for both groups of infants. Infant demographic profiles reflected a sample of low-risk, full-term infants with a fairly equal distribution of boys and girls (control infants, 25 boys and 32 girls; treatment infants, 30 boys and 34 girls). No statistically significant differences between the two groups were found on the demographic variables collected (Keefe et al., 2006).

Sporadic missing data occurred because of parents missing or refusing to answer occasional items on the PSI-SF. A few surveys $(n=16)$ were lost because of attrition from the study. These study dropouts did not differ from the cohort members by mothers' age, infant birth weight, or hours crying. Completion rates were not different for the treatment versus the control group. However, chi-square analysis revealed that the 16 mothers who failed to complete one or more of the surveys were significantly less educated; $26.7 \%$ of these mothers had less than a high school education compared with $7.3 \%$ of those that completed the study, $[\mathrm{chi}]^{2}(1)=5.9, \mathrm{p}=.02$.

At baseline, no significant differences were found in the total PSI-SF scores when comparing the control and treatment groups. However, there was a significant difference $(p=.009)$ in the baseline scores for the DC subscale, with control group scores (mean $=38.2$ ) higher than the treatment group scores (mean $=35.8$ ). This difference was accounted for in the time-bytreatment interaction in the repeated measures ANOVA model, which includes data from all three time points. No pretreatment or posttreatment differences were detected in the two geographically separate study sites; therefore, data were combined for the analysis.

The results of the repeated measures ANOVA for total PSI-SF scores (Table 2) revealed a significant main effect for time in both the treatment and control groups $(p<.001)$. The treatment group experienced a larger decrease in total PSI scores from the 4-week visit to the 8week visit $($ mean $=71.0)$ when compared with the control group $($ mean $=77.2)$, although this 
time-by-treatment interaction was not statistically significant (Figure 3). A significant effect of time-by-treatment was found in the P-CDI subscale $(\mathrm{p}=.04)$; mothers in the treatment group reported a larger decrease in parenting stress related to interactions with the infants between 4 and 8 weeks (17.6) than did those in the control group (20.4; Figure 4). The two other subscales followed a pattern similar to the total PSI-SF score, with a main effect for time and no statistically significant time-by-treatment interactions (Figures 5 and 6).

\begin{tabular}{llccc} 
Outcome & \multicolumn{1}{c}{ Effect } & $\boldsymbol{d f}$ & $\boldsymbol{F}$ & $\boldsymbol{p}$ \\
\hline PSI total stress & Time & 2,119 & 41.6 & $<.001$ \\
& Treatment & 1,119 & 2.3 & .13 \\
& Time-by-treatment $^{2,119}$ & 1.6 & .20 \\
& Treatment at posttest $^{\star}$ & 2,160 & 20.8 & $<.001$ \\
\hline
\end{tabular}

\section{Notes. ANOVA $=$ Analysis of Variance; PSI = Parenting Stress Index. *One-way ANOVA at posttest-only.}

TABLE 2. Repeated Measures ANOVA Comparing Mothers in the Treatment and Control Groups on Parenting Stress 


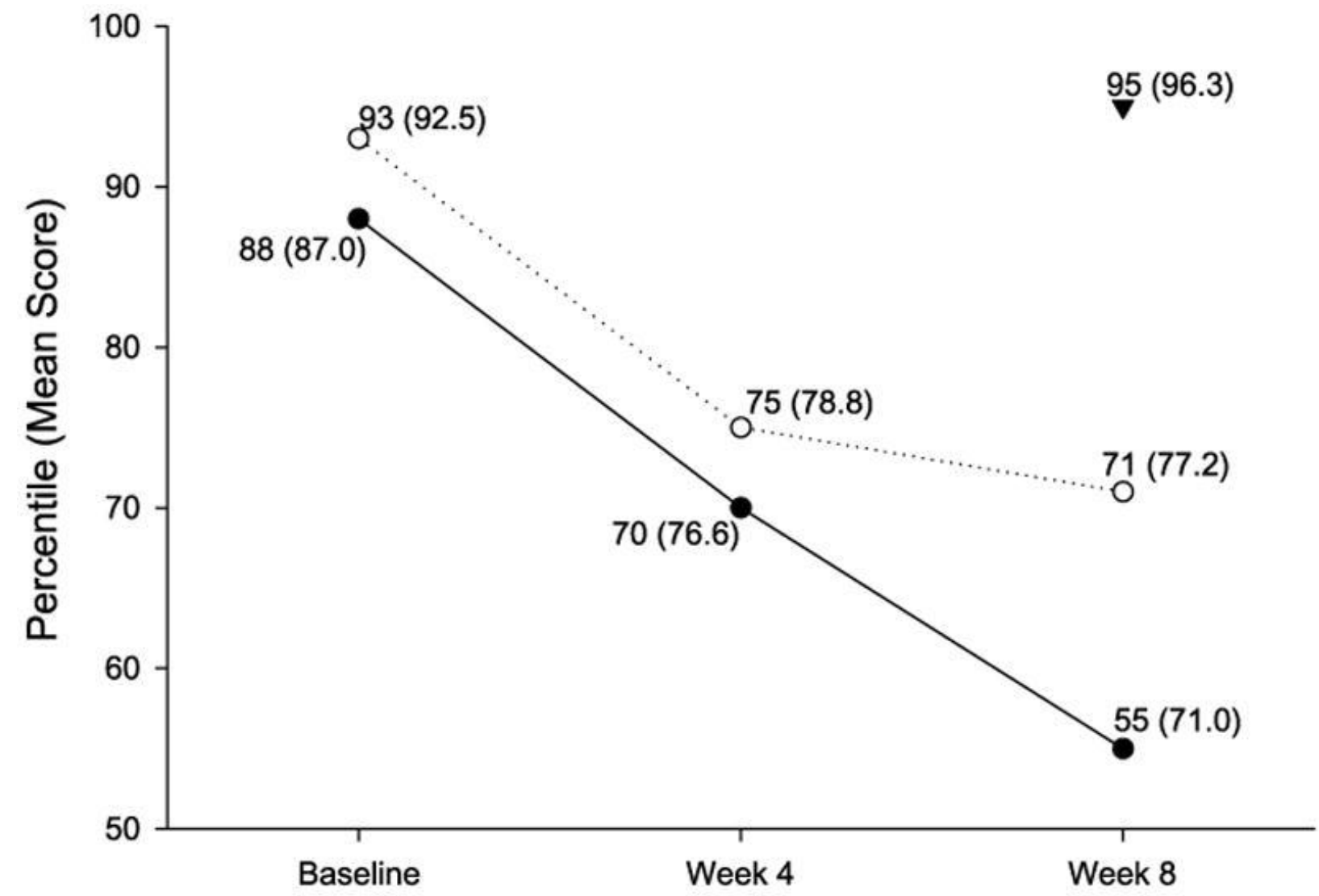

FIGURE 3. PSI-SF total scores by group. Notes., Experimental;, Control;, Posttest. 


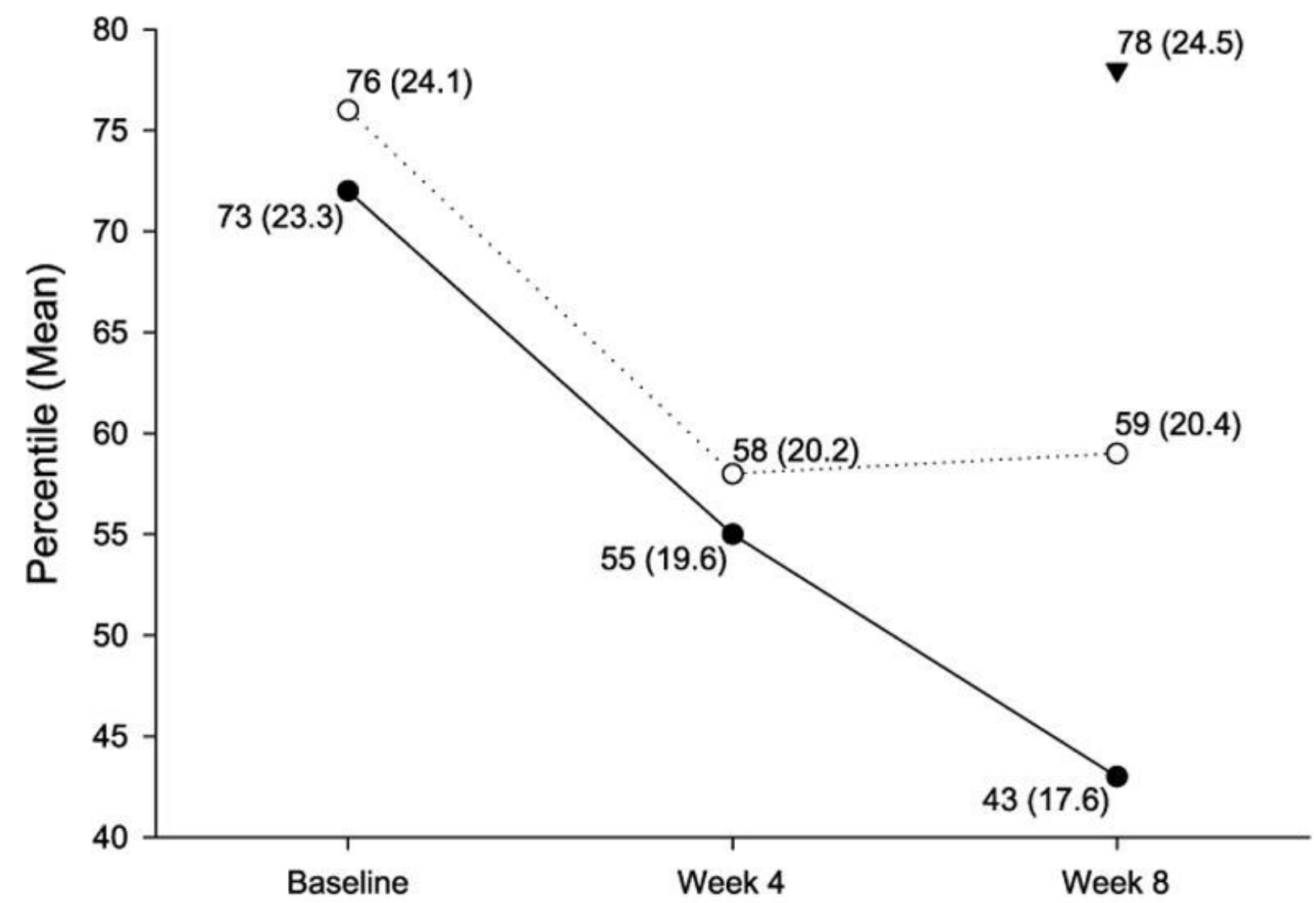

FIGURE 4. PSI-SF parent-child dysfunctional interaction by group. Notes., Experimental;(100\%,p), Control;, Posttest. 


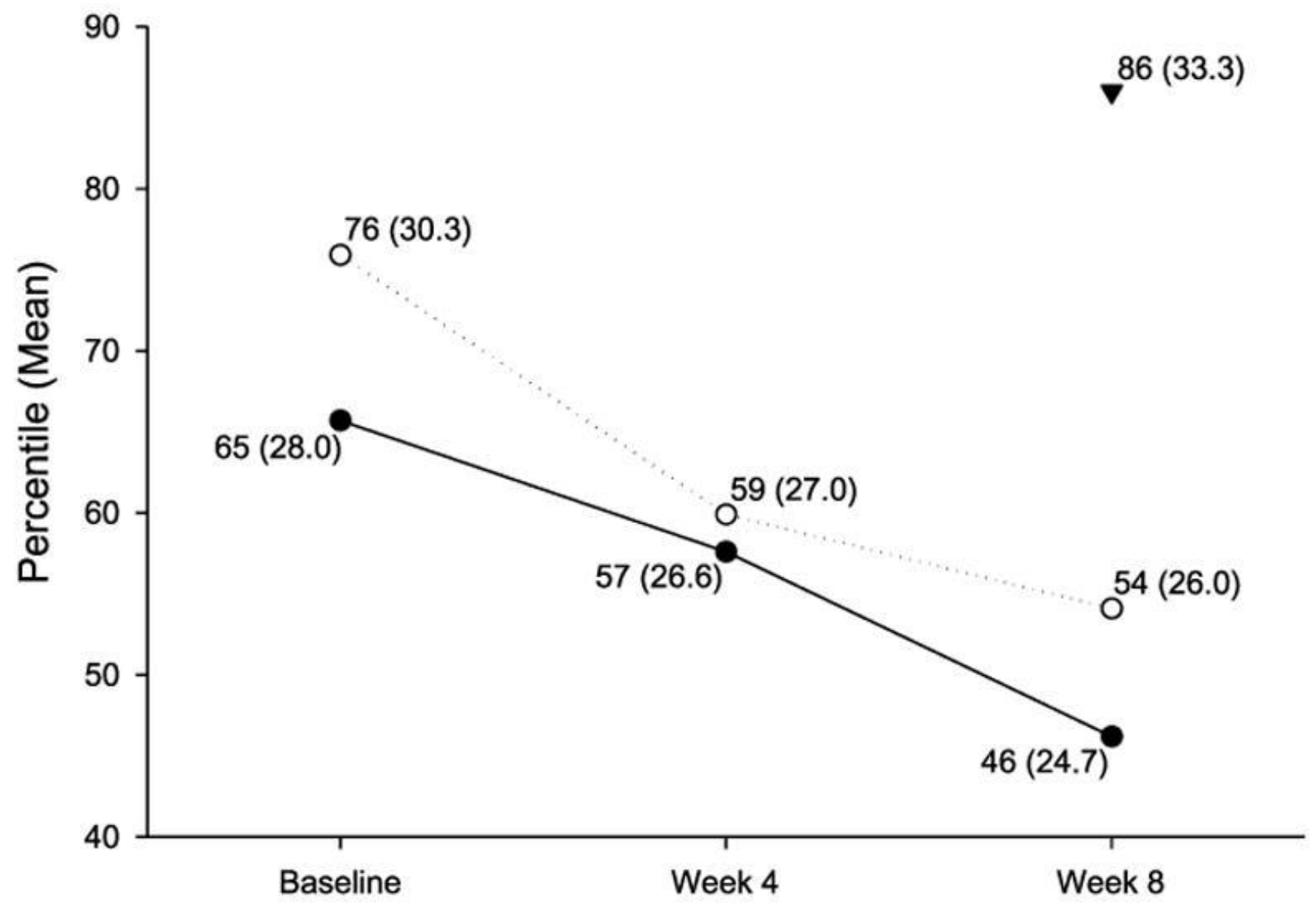

FIGURE 5. PSI-SF parental distress by group. Notes. (100\%,p), Experimental; (100\%,p), Control; (100\%,p), Posttest. 


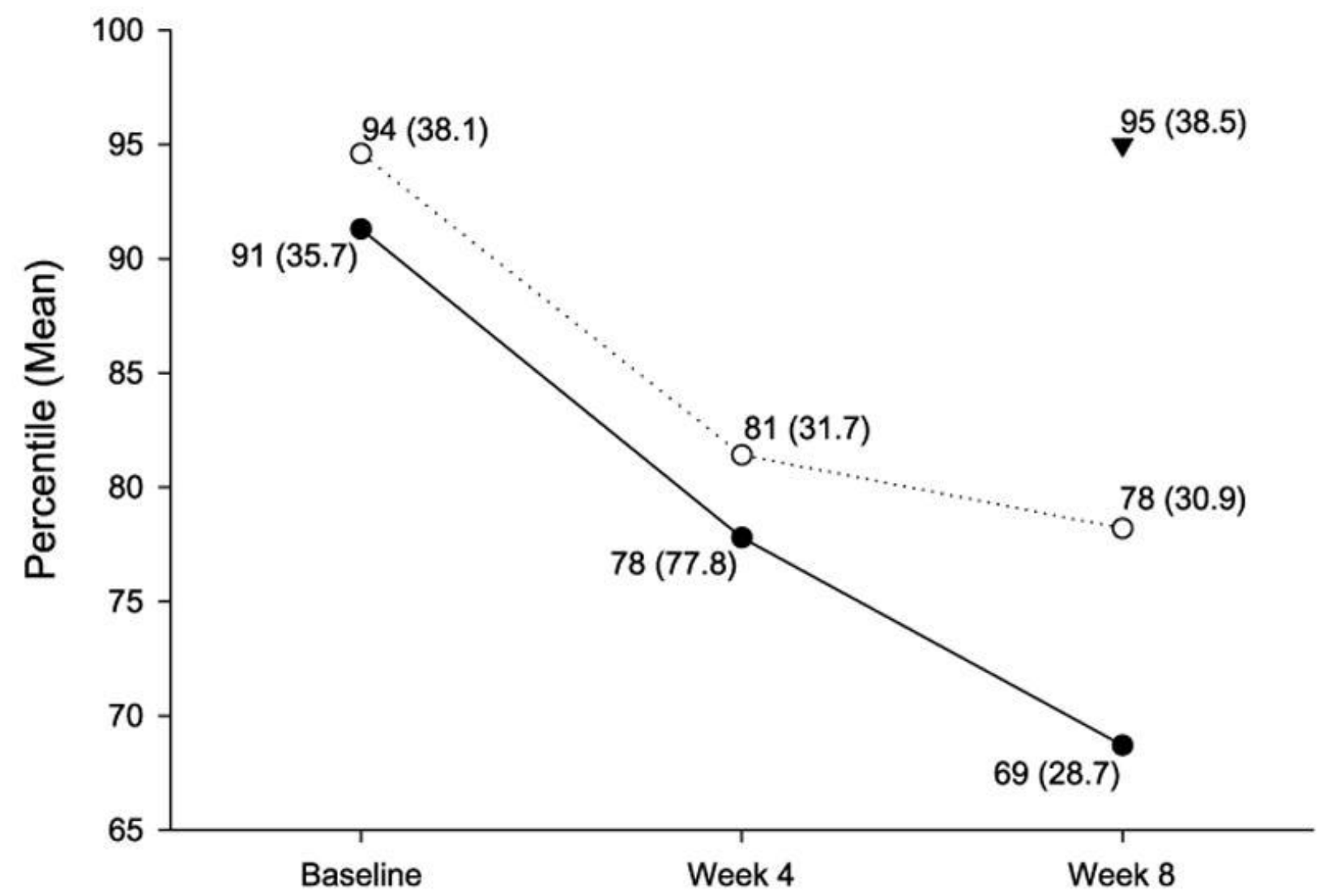

FIGURE 6. PSI-SF difficult child by group. Notes. (100\%,p), Experimental; (100\%,p), Control; $(100 \%, p)$, Posttest.

At the exit interviews, parents reported that the nurse visits for data collection and the intervention visits were helpful in reducing the stress level associated with parenting these irritable infants. Several parents in the control group stated that they appreciated the interest and the acknowledgement that they were dealing with a difficult situation and a parenting challenge. They found the intake interview and exit interview questions informative and the focus on sleeping, crying, and interaction patterns interesting. The treatment group received, in addition, four visits from a trained advanced practice nurse who delivered the REST Routine intervention program to families in their homes. To test the hypothesis that the control groups derived some benefit from the nurse visits for data collection and the Hawthorne effect of participating in the study, a third group $(n=43)$ of infants too old at entry for randomization (mean age $=10.4 \pm 2.1$ weeks) was enrolled and analyzed as a posttest-only group. This posttest-only group was measured only once (postintervention) and was used to assess the influence of an unintended treatment effect in the control group. These parents reported statistically significantly higher levels of total parenting stress (Figures 2-5), which showed up in all three subscales $(\mathrm{p}<.001)$. Although these findings are interesting, they should not be overinterpreted because these infants were not part of the original randomization design. The age of the infants and other factors not assessed may render them not comparable.

The percentile rankings from this study of mothers of irritable infants revealed a high level of initial parenting stress. Almost half $(45.5 \%)$ of the mothers completing the questionnaire at baseline reported a parenting stress level that was equal to or greater that the 90th percentile for 
the normative dataset. Persistently high levels of perceived difficulty in the infants were noted in this study, as compared with the standardized normative rankings. At the 8-week follow-up visit, the DC scores remained at the 78th and 69th percentiles for the control and treatment groups, respectively. Although not statistically significant, total parenting stress level for the parents in the treatment group had decreased to the 54th percentile, and the control group scores were at the 71st percentile.

\section{Summary and Discussion}

In this clinical trial, the parenting stress level was assessed in two groups of randomized irritable infants and one group of posttest-only infants. The first two groups were measured three times over a 2-month time period. Initial total parenting stress levels were alarmingly high (greater than 85th percentile) in all three groups: treatment, control, and posttest-only. At the 8-week follow-up visit, the parents of the posttest-only group of infants reported significantly higher levels of total parenting stress and in all three areas assessed by the subscales. These data provide further evidence of the elevated stress levels associated with parenting an irritable infant. The home-based nursing intervention was not shown to be effective in reducing total parenting stress. However, the parenting stress related to parent-child interaction was significantly reduced. Scores for parents in the treatment group revealed statistically significant improvement in this subscale, which is designed to assess parents' perception of whether their child does or does not meet expectations and whether interactions with the child are reinforcing (Reitman et al., 2002).

The program provided structured reassurances about their parenting competence, empathetic listening, support, and permission for time-out for the parents. Also incorporated were the use of infant behavior assessment, state regulation recommendations, individualized daily schedules to provide structure and routine, specific management strategies to reduce environmental overload, and information on soothing practices and positions. The findings suggest that these strategies may be helpful in improving the reinforcing value and quality of interaction for these parents. Parental distress and perception of difficult behaviors in the child improved in the treatment group, but not with statistical significance. Although some data trends are interesting, they did not all reach statistical significance, in part because of the limitations of the sample size and the variability to detect mean differences in the groups. The sample size for this study was originally calculated at 80 participants per group to be able to detect an overall effect size of .64. However, because of a reduction in the budget and timeline, this goal was not achieved for the two randomized groups alone. Selection criteria relating to the age of the infant at intake also limited the participants eligible for the two original cohorts, leading to the decision to add the third group. Another limitation related to the sample characteristics is that the generalizability is best restricted to middle and upper-middle, educated, White mothers and infants. Further studies are needed to extrapolate these findings to other groups and to evaluate the essential components and the cost-benefit ratio.

This research is based on the premise that it is important to identify parent-infant dyads at-risk for dysfunctional parent-child relationships and to use appropriate interventions to improve these relationships. The PSI-SF was found to be useful in assessing parenting stress related to caring for an irritable infant over time. The findings indicate that parenting stress is high in parents with irritable or colicky infants, and this stress level decreased over time. The overall effectiveness of 
a home-based nursing intervention program was difficult to evaluate because the nursing visits to the home were found to be useful and supportive even if intended only for data collection.

\section{Acknowledgments}

Funded by a grant from the National Institute of Nursing Research (R01-NR04661)

\section{References}

Abidin, R. R. (1995). Parenting Stress Index professional manual (3rd ed.). Odessa, FL:

Psychological Assessment Resources.

Barr, R. G., St. James-Roberts, I., \& Keefe, M. R. (2001a). Early infant crying: A parent's guide. New Brunswick, NJ: Johnson \& Johnson Consumer Companies.

Barr, R. G., St. James-Roberts, I., \& Keefe, M. R. (2001b). New evidence on unexplained early crying: Its origins, nature and management. New Brunswick, NJ: Johnson \& Johnson Consumer Companies.

Brazelton, T. B. (1995). Introduction. In T. B. Brazelton \& J. K. Nugent (Eds.), Neonatal Behavioral Assessment Scale (3rd ed., pp. 15-18). London: Mac Keith Press.

Butz, A. M., Pulsifer, M., Marano, N., Belcher, H., Lears, M. K., \& Royall, R. (2001). Effectiveness of a home intervention for perceived child behavioral problems and parenting stress in children with in utero drug exposure. Archives of Pediatrics \& Adolescent Medicine, 155, 1029-1037.

Hiscock, H., \& Wake, M. (2002). Randomized controlled trial of behavioral infant sleep intervention to improve infant sleep and maternal mood. BMJ, 324, 1062-1067.

Humphrey, R. A., \& Hock, E. (1989). Infants with colic: A study of maternal stress and anxiety. Infant Mental Health Journal, 10, 263-272.

Keefe, M. R. (1988). Irritable infant syndrome: Theoretical perspectives and practice implications. Advances in Nursing Science, 10(3), 70-78.

Keefe, M. R., Barbosa, G., Froese-Fretz, A., Kotzer, A. M., \& Lobo, M. (2005). An intervention program for families with irritable infants. American Journal of Maternal Child Nursing, 30(4), 230-236.

Keefe, M. R., \& Froese-Fretz, A. (1991). Living with an irritable infant: Maternal perspectives. American Journal of Maternal Child Nursing, 16(5), 255-259.

Keefe, M. R., Froese-Fretz, A., \& Kotzer, A. M. (1997). The REST regimen: An individualized nursing intervention for infant irritability. American Journal of Maternal Child Nursing, 22(1), 16-20.

Keefe, M. R., Lobo, M., Froese-Fretz, A., Kotzer, A. M., Barbosa G., \& Dudley, W. (2006). Effectiveness of an intervention for colic. Clinical Pediatric, 45(2), 123-134.

Murray, L., \& Cooper, P. (2001). The impact of irritable infant behavior on maternal mental state: A longitudinal study and a treatment trial. In R. G. Barr, I. St. James-Roberts, \& M. R. Keefe (Eds.), New evidence on unexplained early crying: Its origins, nature and management (pp. 149-164). New Brunswick, NJ: Johnson \& Johnson Consumer Companies.

Papousek, M., \& von Hofacker, N. (1998). Persistent crying in early infancy: A non-trivial condition of risk for the developing mother-infant relationship. Child: Care, Health and Development, 24(5), 395-424.

Reitman, D., Currier, R. O., \& Stickle, T. R. (2002). A critical evaluation of the Parenting Stress Index-Short Form (PSI-SF) in a head start population. Journal of Clinical Child and Adolescent Psychology, 31(3), 384-392. 
St. James-Roberts, I., \& Plewis, I. (1996). Individual differences, daily fluctuations, and developmental changes in amounts of infant waking, fussing, crying, feeding, and sleeping. Child Development, 67, 2527-2540.

St. James-Roberts, I., Sleep, J., Morris, S., Owen, C., \& Gillham, P. (2001). Use of a behavioral program in the first 3 months to prevent infant crying and sleeping problems. Journal of Paediatrics and Child Health, 37(3), 289-297.

Stifter, C. A., \& Bono, M. A. (1998). The effect of infant colic on maternal self-perceptions and mother-infant attachment. Child: Care, Health and Development, 24(5), 339-351.

Thomas, K. A., Renaud, M. T., \& DePaul, D. (2004). Use of the parenting stress index in mothers of preterm infants. Advances in Neonatal Care, 4(1), 33-41.

Thompson, P. E., Harris, C. C., \& Bitowski, B. E. (1986). Effects of infant colic on the family: Implications for practice. Issues in Comprehensive Pediatric Nursing, 9(4), 273-285.

Uzark, K., \& Jones, K. (2003). Parenting stress and children with heart disease. Journal of Pediatric Health Care, 17(4), 163-168.

Youngblut, J. M., Brooten, D., Singer, L. T., Standing, T., Lee, H., \& Rodgers, W. L. (2001). Effects of maternal employment and prematurity on child outcomes in single parent families. Nursing Research, 50(6), 346-355. 